\title{
Improving urgent and emergency care in England
}

\author{
Jonathan R Benger national clinical director for urgent care, Keith Willett director for acute episodes \\ of care (domain 3)
}

NHS England, Leeds LS2 7UE, UK

Roland and Boyle's editorial on Keogh's review of urgent and emergency care is welcome but contains several inaccuracies. ${ }^{1}$ The review is informed by a detailed consideration of the available evidence (appendix 1), which does not ignore the need to reduce hospital bed occupancy or relevant evidence from the King's Fund. ${ }^{2}$ Neither does the report "curiously omit" the option to colocate primary care centres adjacent to emergency departments (proposal can be found on page 25). We also recognise the important role that social care plays in facilitating discharge and preventing hospital admission, and this is being dealt with through the integrated care and support programme. ${ }^{3}$

We agree that frail and older people present an important challenge to the system, which is why the review advocates a comprehensive community based approach, supported by specialist expertise, to avoid hospital admission wherever possible. Similarly, the report sets out a clear plan for progressively developing 111 and for providing the senior clinical input that Roland and Boyle recommend.

Roland and Boyle's suggestions for improvement are welcome, and we encourage further contributions from patients and clinicians (www.nhs.uk/NHSEngland/keogh-review/Pages/ about-the-review.aspx). ${ }^{4}$

Competing interests: Both authors are employed by NHS England, contributed to the phase one report, and are actively engaged in the delivery of the urgent and emergency care review.

1 Roland M, Boyle AA. Urgent care in England. BMJ 2013;347:f7046. (25 November.)

2 NHS England. Transforming urgent and emergency care services in England. Urgent and emergency care review. End of phase 1 report. Appendix 1—revised evidence base from the urgent and emergency care review. 2013. www.nhs.uk/NHSEngland/keogh-review/ Documents/UECR.Ph1Report.Appendix\%201.EvBase.FV.pdf.

3 Department of Health. Integrated care and support: our shared commitment. 2013. https: //www.gov.uk/government/uploads/system/uploads/attachment_data/file/198748/ DEFINITIVE_FINAL_VERSION_Integrated_Care_and_Support_-_Our_Shared Commitment_2013-05-13.pdf.

4 NHS England. Transforming urgent and emergency care services in England. Urgent and emergency care review end of phase 1 report. Appendix 3-summary of engagement responses. 2013. www.nhs.uk/NHSEngland/keogh-review/Documents/UECR.Ph1Report. Appendix\%203.Engage.Results.FV.pdf.

Cite this as: BMJ 2013;347:f7495

๑ BMJ Publishing Group Ltd 2013 